\title{
ACTIVE CONTROLS IN INTERFEROMETRIC DETECTORS OF GRAVITATIONAL WAVES: INERTIAL DAMPING OF THE VIRGO SUPERATTENUATOR*
}

\author{
GIOVANNI LOSURDO \\ Istituto Nazionale di Fisica Nucleare - Sezione di Pisa \\ E-mail: losurdo@galileo.pi.infn.it
}

\begin{abstract}
The operation of an interferometer for gravitational waves detection requires sophisticated feedback controls in many parts of the apparatus. The aim of this lecture is to introduce the types of problems to be faced in this line of research. The attention is focused on the inertial damping of the test mass suspension of the VIRGO interferometer (the superattenuator): it is a multidimensional local control aimed to reduce the residual motion of the suspended mirror associated to the normal modes of the suspension. Its performance is very important for the locking of the interferometer.
\end{abstract}

\section{Introduction}

Operating an interferometer for gravitational waves detection requires the implementation of many active controls on the different parts of the apparatus. For instance, feedback controls are needed to reduce the laser frequency and power fluctuations, to keep the optical cavities in resonance and to maintain the interferometer output on a dark fringe. The basic idea is that the apparatus works at its best strictly around a well defined working point 4 (laser fluctuations and shot noise at a minimum, optical cavities in resonance and interferometer output on a dark fringe). Internal and external disturbances overload the dynamic range of the interferometer. Therefore, the system has to be forced to remain in the correct working position. This is achieved via feedback controls.

In this lecture we examine in detail one of the controls needed to reach the final goal of operating the interferometer: the inertial damping of the VIRGO superattenuator (SA) 3. The SA can be described as a chain of mechanical "filters", each one acting as a "spring" in 6 degrees of freedom. The normal mode frequencies of the SA range between 0.04 and about $2 \mathrm{~Hz}$. At frequencies $f>>2 \mathrm{~Hz}$ the $\mathrm{SA}$ acts as a steep filter of the seismic vibrations of the ground (an attenuation factor of $10^{15} @ 10 \mathrm{~Hz}$ is expected). Therefore,

\footnotetext{
*LECTURE GIVEN AT THE INTERNATIONAL SUMMER SCHOOL ON EXPERIMENTAL PHYSICS OF GRAVITATIONAL WAVES - URBINO (ITALY), SEPTEMBER 6-18, 1999
} 
in the interferometer detection band (10 Hz-few $\mathrm{kHz})$, the suspended mirror is "disconnected" from the ground. Beside being a low pass filter for ground vibrations the SA is a tool for actively controlling the mirror position. Forces that move and steer the pirror can be exerted in 3 points of the SA chain: the inverted pendulum (IP) $\mathrm{E}$, the marionetta (a special mechanical tool designed to steer the mirror) and the mirror itself (from a suspended reference mass).

Keeping the interferometer in the operating position requires the mirror to have a maximum RMS relative motion of less the $10^{-12} \mathrm{~m}$. In the frequency range where the SA is fully effective $(f>>4 \mathrm{~Hz})$ the residual motion of the mirror is negligible. On the other hand, the mirror free motion in the region of the SA normal modes is $\sim 100 \mu \mathrm{m}$. Feedback forces acting on the SA must reduce the mirror motion from $100 \mu \mathrm{m}$ to $10^{-12} \mathrm{~m}$. The dynamic range of a feedback system able to perform this control has to be huge: the control is performed in three steps (hierarchical control 2). The first step is a damping of the SA normal modes in order to reduce the mirror residual motion to less than $10 \mu \mathrm{m}$. This is necessary in order to control the mirror position acting on the lower stages without reinjecting noise into the detection band. We describe in the following an implementation of a high gain and wideband damping of the SA resonances.

\section{Experimental setup}

The setup (fig. 1) of the experiment is composed of a full scale superattenuator, provided with 3 accelerometers (placed on the top of the IP), 3 LVDT position sensors (measuring the relative motion of the IP with respect to an external frame), 3 coil-magnet actuators. The accelerometers work in the range DC-400 Hzand have acceleration spectral sensitivity $\sim 10^{-9} \mathrm{~m} \mathrm{~s}^{-2} \mathrm{~Hz}^{-1 / 2}$ below $3 \mathrm{~Hz}$. The sensors and actuators are all placed in pin-wheel configuration. The sensor and actuator signals are computer controlled by a ADC (16 bit)-DSP-DAC (20 bit) system. The DSP handles the signals of all the sensors and actuators. It can combine them by means of matrices, create complex feedback filters (like the one of fig. 8) with high precision poles/zeroes placement and perform all the calculations at a high sampling rate $(10 \mathrm{kHz})$. The suspended mirror is also provided with LVDT position sensors to measure its displacement with respect to ground.

\section{The approach to the problem of control}

The IP, where all the sensors are placed and on which the forces are exerted, has 3 main resonant modes: two translation $(X, Y)$ and the rotation around

paper: submitted to World Scientific on January, 7, 2000 

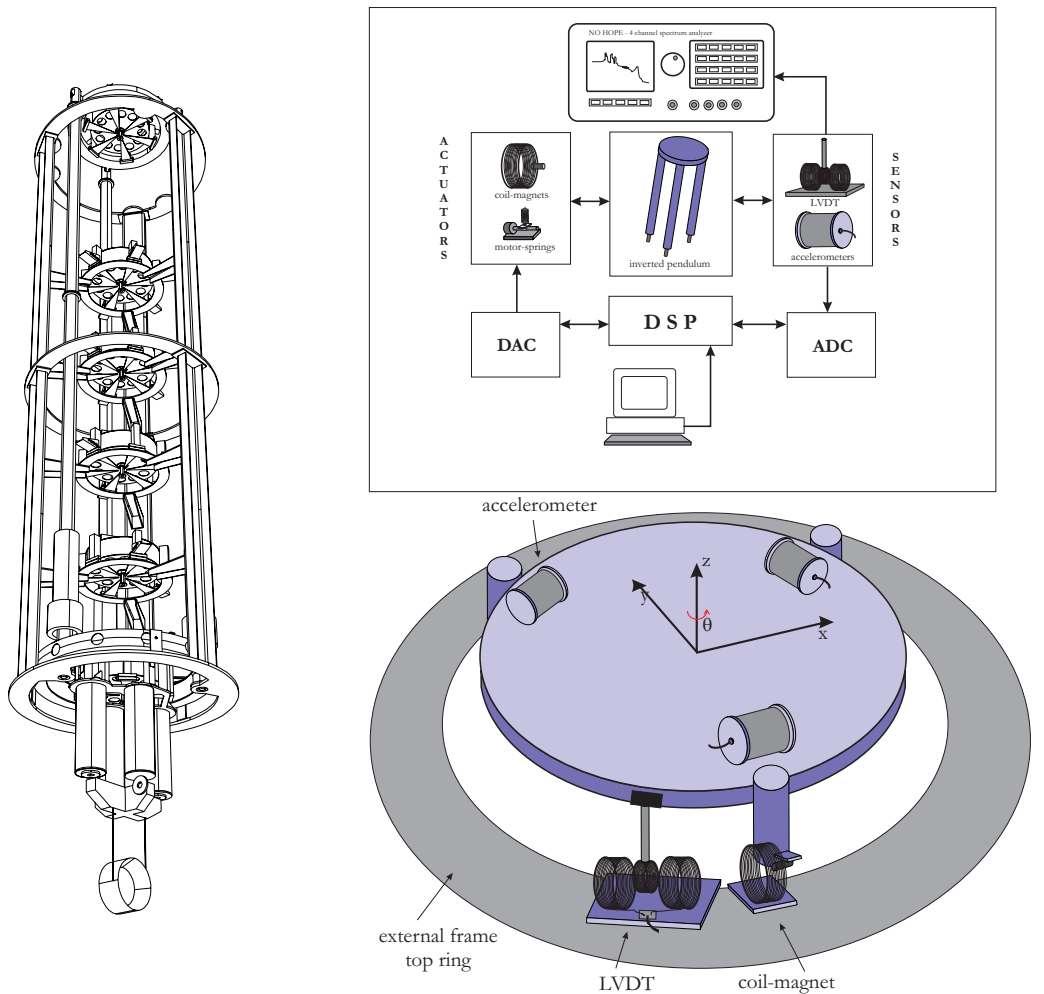

Figure 1. LEFT: the superattenuator; RIGHT TOP: logical scheme of the setup for the local active control; RIGHT BOTTOM: simplified view of the IP top table, provided with the 3 accelerometers. One LVDT position sensors and one coil-magnet actuator are also shown.

the vertical axis $(\Theta)$. Each sensor is sensitive to all the modes and each actuator can excite all the modes. In control theory language, such a system is defined MIMO (multiple in-multiple out). Controlling a MIMO system can be very difficult. Our approach has been different: the signals of the 3 sensors are digitally mixed (using proper transformation matrices) to build up virtual sensors, sensitive to one mode only and "blind" to the others. At the same time, we build up virtual actuators able to excite each mode separately. The system is thus uncoupled into 3 SISO (single in-single out) subsystems. In terms of analytical mechanics this means the system is described in the normal 


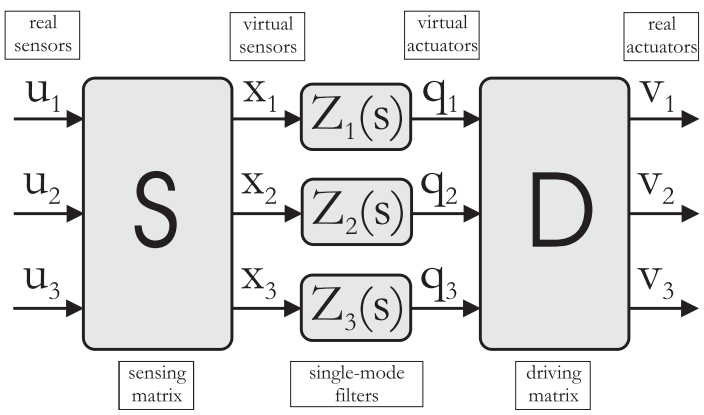

Figure 2. The logic of the diagonalisation: the output $u_{i}$ of the sensors are linearly combined by a matrix $\mathbf{S}$ in order to produce 3 virtual sensors outputs $\left(x_{i}\right)$, sensitive to pure modes. Three independent feedback filters $Z_{i}(s)$ are designed for the pure modes and 3 generalized forces $q_{i}$ are produced. The $q_{i}$ are turned into real currents $\left(v_{i}\right)$ to feed the actuators via the matrix $\mathbf{D}$.

modes basis. The equations of motions take the form:

$$
\ddot{x}_{k}+\omega_{k}^{2} x_{k}=q_{k} \quad, \quad k=1 \ldots 3
$$

where $x_{k}$ is the $k$-th normal mode, $\omega_{k} / 2 \pi$ is the corresponding resonant frequency and $q_{k}$ the generalized force on that mode. Let $\mathbf{u}=\left(u_{1}, \ldots, u_{3}\right)$ the vector made by the outputs of the 3 sensors and let $\mathbf{x}=\left(x_{1}, \ldots, x_{3}\right)$ the vector made by the 3 virtual sensors. Analogously, let $\mathbf{v}$ the vector made by the 3 currents driving the actuators and $\mathbf{q}$ the vector of the 3 generalized forces. The transformation from the system of the physical sensors/actuators to that of the virtual ones is operated by two matrices, such that:

$$
\begin{aligned}
& \mathbf{v}=\mathbf{D q} \\
& \mathbf{x}=\mathbf{S u}
\end{aligned}
$$

The sensing $(\mathbf{S})$ and driving $(\mathbf{D})$ matrices are experimentally measured. The measurement procedure is described in details in ref. 9. The result of the measurement is determined by the mechanical characteristics of the system (resonant frequencies, quality factors), the geometry of the sensors and actuators and their calibration, but it is not needed to know them in order to measure the matrices. The logic of diagonalisation is explained by fig. 2 .

After the diagonalisation the system can be considered as composed of 3 uncoupled oscillators, and the control strategy for each of them has to be defined independently (see fig. 3). 

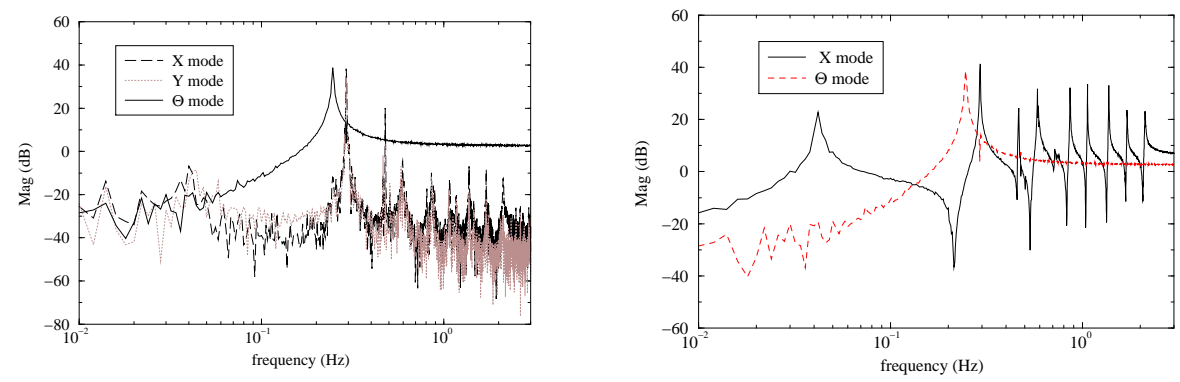

Figure 3. Effect of the digital diagonalisation. LEFT: the output of the 3 virtual accelerometers when $\Theta$ is excited. RIGHT: the output of the virtual accelerometers $X$ and $\Theta$ are compared. Different feedback strategies are required in the two cases, because $X$ senses all the translational modes of the SA chain.

\section{Inertial damping: principle}

The control we describe here is called inertial damping because it is performed by using (mostly) inertial sensors (accelerometers). In the following, with the help of a simple model, we explain why this is the best choice to achieve a high performance damping.

Let us consider a simple pendulum of mass $m$ and length $l$. Let $x$ be the abscissa of the suspended mass, $x_{0}$ that of the suspension point. Let $F_{\mathrm{fb}}$ the external force on the pendulum (i.e. the feedback force to control it). The equation of motion is then:

$$
F_{\mathrm{fb}}=m \ddot{x}+\gamma \dot{x}+k\left(x-x_{0}\right)
$$

where $\gamma$ is the viscous dissipation factor and $k=m g / l$. The control loop of

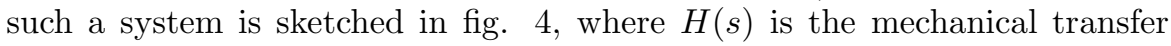
function, $G(s)$ is the compensator and out is the output of the sensor used. The goal of the control is to damp the pendulum resonance. This can be done easily with a viscous (theoretical) feedback force:

$$
F_{\mathrm{fb}}=-\gamma^{\prime} \dot{x}
$$

Our sensors do not measure $x$. Their output is:

$$
\text { out }= \begin{cases}x-x_{0} & \text { for displacement sensors } \\ \ddot{x} & \text { for accelerometers }\end{cases}
$$

Therefore, the actual "viscous" force that can be built if position sensors are 

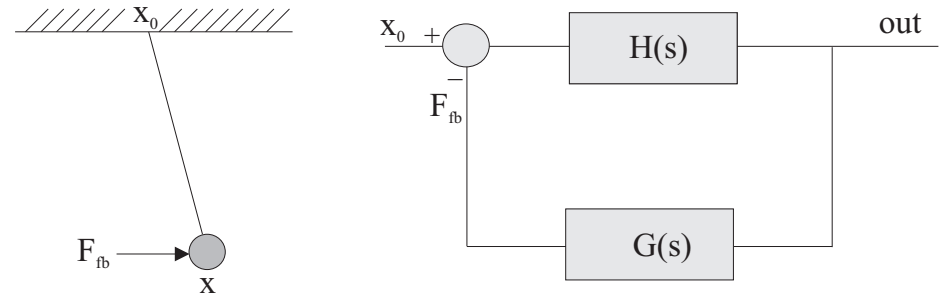

Figure 4. The control scheme for a simple pendulum

used has the form:

$$
F_{\mathrm{fb}}^{p}=-\gamma^{\prime} \frac{\mathrm{d}}{\mathrm{d} t}\left(x-x_{0}\right)
$$

It can be easily shown that with such a feedback force the closed loop equation of motion (in Laplace space) reduces to:

$$
x(s)=\frac{\omega_{0}^{2}+G_{0} s}{s^{2}+\omega_{0}^{2}+\left(\omega_{0} / Q+G_{0}\right) s} \cdot x_{0}(s)
$$

where $G_{0}=\gamma^{\prime} / m$ is a gain parameter $a^{a}$ measuring the intensity of the viscous feedback force, and $Q$ is the open loop quality factor. When the loop is closed a damping of the resonance is achieved:

$$
Q^{\prime} \stackrel{G>1}{\longrightarrow} \frac{\omega_{0}}{G}
$$

Nevertheless, as the gain is increased, a larger amount of noise is reinjected off-resonance. This is associated to the term " $G_{0} s$ " in the numerator of (8) and depends on the fact that the sensor used to build up the feedback force measures the position of the pendulum with respect to ground. Therefore, an infinitely efficient feedback would "freeze" the pendulum to ground (which is seismic noisy), reducing its motion at the resonance, with the drawback of bypassing its attenuation properties above resonance.

The situation is fairly different when an inertial sensor is used. In this case the viscous feedback force is obtained by integrating the accelerometer output, and the output does not depend on $x_{0}$ :

$$
F_{\mathrm{fb}}^{a}=-\gamma^{\prime} \int \ddot{x} \mathrm{~d} t
$$

${ }^{a}$ In a real feedback system a frequency dependent gain function $G(s)$ rather than a gain parameter has to be considered. 

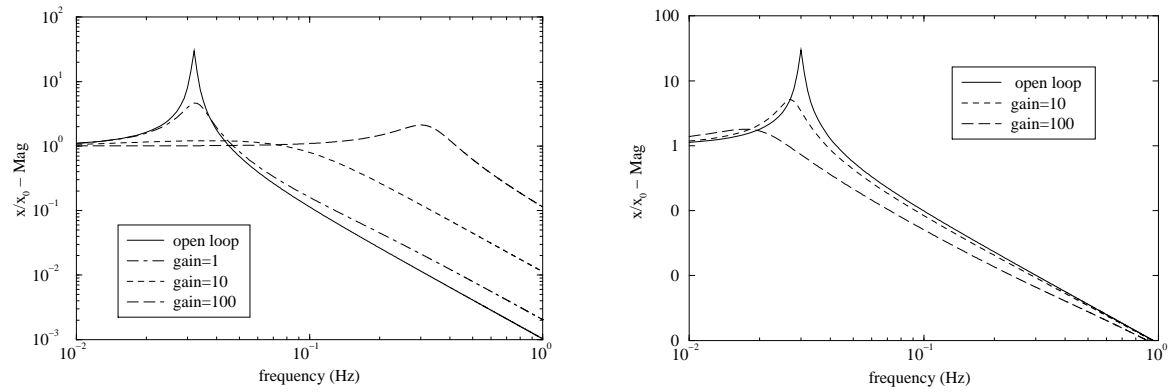

Figure 5. Damping of a simple pendulum: the closed loop transfer function $x(s) / x_{0}(s)$ (magnitude) when a position sensor is used (LEFT) and when an accelerometer is used (RIGHT).

The closed loop equation of motion is then:

$$
x(s)=\frac{\omega_{0}^{2}}{s^{2}+\omega_{0}^{2}+\left(\omega_{0} / Q+G_{0}\right) s} \cdot x_{0}(s)
$$

A damping of the resonance is obtained (exactly as in the previous case) but without reinjection of off-resonance noise. In fig. 5 a simulation of the closed loop transfer function $x(s) / x_{0}(s)$ is shown in the two cases. Up to now we have considered a simple viscous damping. It is possible to increase the bandwidth of the control if the feedback force contains a term proportional to $x$ (the double integral of the accelerometer signal). The result obtained in this case is shown in fig. 6 .

\section{Control strategy}

In this section we extend the principles of the previous section and describe the strategy to control the SA.

The basic idea of inertial damping is to use the accelerometer signal to build up the feedback force. As a matter of fact, an infinitely efficient feedback using only the inertial sensor information, would null the acceleration of the pendulum, but it would not do anything if the pendulum moves at constant velocity. Such a control would be unstable with respect to drifts. Therefore, if the control band is to be extended down to DC, a position signal is necessary. Our solution was a merging of the two sensors: the virtual LVDT (position)

and accelerometer signals are combined in such a way that the LVDT signal $(l(s))$ dominates below a chosen cross frequency $f_{\text {merge }}$ while the accelerometer 


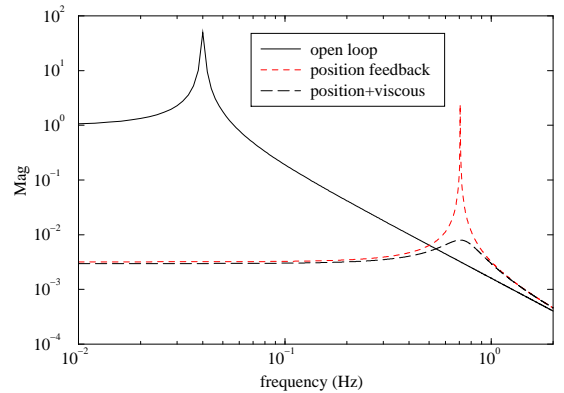

Figure 6. Inertial damping of a simple pendulum when a position feedback is implemented.

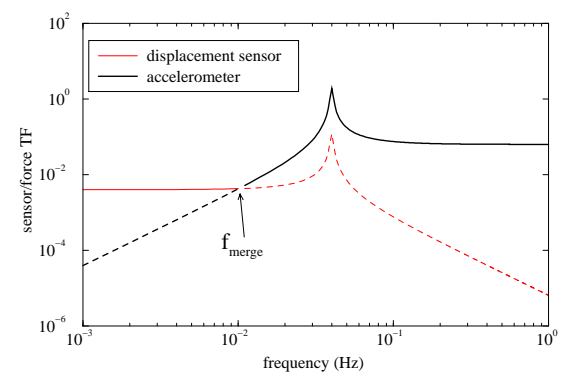

Figure 7. Merging of displacement and acceleration sensors (simulation for a simple pendulum)

signal $(a(s))$ dominates above it (see fig. 7 and ref. 7 ). The feedback force has the form

$$
F_{\mathrm{fb}}=G(s)[a(s)+\epsilon l(s)]
$$

where $G(s)$ is the digital filter transfer function (see fig. 8) and $\epsilon$ is the parameter whose value determines $f_{\text {merge }}$. We have chosen $f_{\text {merge }} \sim 10 \mathrm{mHz}$ (corresponding to $\epsilon \sim 5 \cdot 10^{-3}$ ). This approach stabilizes the system with respect to low frequency drifts at the cost of reinjecting a fraction $\epsilon$ of the seismic noise via the feedback.

We describe in the following the feedback design for the 3 d.o.f., starting from the the translational ones. The virtual $X$ and $Y$ sensors show many resonant peaks (the modes of a chain of pendulums) and this requires a more sophisticated feedback strategy. The digital filter used to control the translation modes $(G(s))$ is shown in fig. 8 (LEFT). It shows three main features:

- for $0.01<f<2 \mathrm{~Hz}$ the gain is proportional to $f^{-2}$. This corresponds to the case of fig. 6: the accelerometer signal is integrated twice and the feedback force is proportional to $x$;

- for $f>2 \mathrm{~Hz}$ the gain is proportional to $f^{-1}$. The accelerometer signal is integrated once: the feedback force is proportional to the velocity and a viscous damping is achieved;

${ }^{b}$ Actually, the LVDT signal $l(s)$ is properly filtered in order to preserve feedback stability at the crossover frequency and in order to reduce the amount of reinjected noise at $f>f_{\text {merge }}$. 

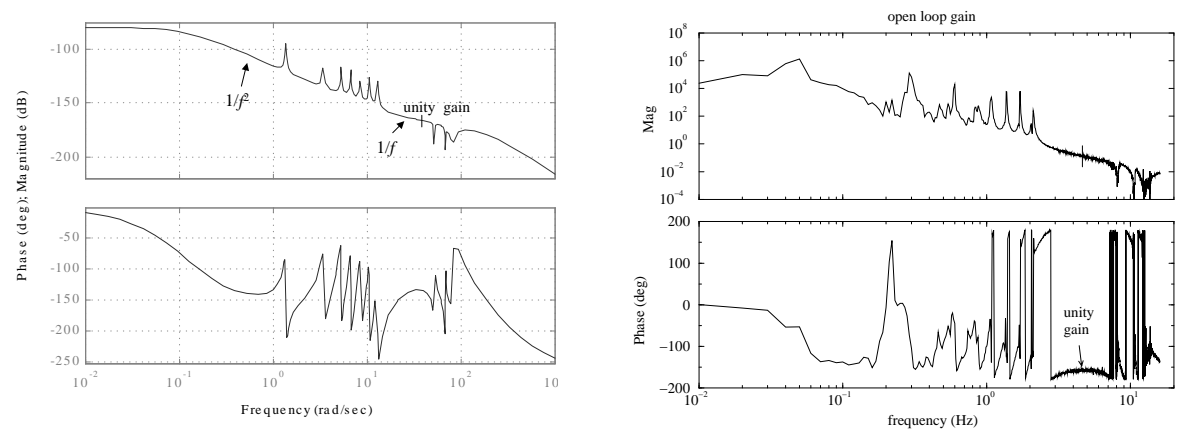

Figure 8. LEFT: Digital filter used for the inertial damping of a translation mode $(X)$. The filter slope is $f^{-2}$ in the range $10 \mathrm{mHz}<f<3 \mathrm{~Hz}, f^{-1}$ for $f>3 \mathrm{~Hz}$. The unity gain is at 4 $\mathrm{Hz}$. The peaks in the digital filter are necessary to compensate the dips in the mechanical transfer function (see the transfer function of the $X$ mode in fig. B). RIGHT: open loop gain function (measured). The phase margin at the unity gain frequency is about $25^{\circ}$.

- the peaks visible in the filter are necessary to compensate the corresponding dips in the mechanical transfer function $(H(s))$ of fig. 3, in order to make the feedback stable.

Fig. 8 (RIGHT) shows the open loop gain transfer function $G(s) H(s)$.

The damping strategy for the $\Theta$ mode is simpler: the $\Theta$ virtual sensor (fig. 3, RIGHT) shows one resonance peak only and no dips: no compensation is necessary. Apart from this, the feedback strategy is similar to the ones used for the translational modes.

\section{Inertial damping: experimental results}

The result of the inertial control (on 3 d.o.f.) is shown in figure 9. The measurement has been performed in air. The noise on the top of the IP is reduced over a wide band $(10 \mathrm{mHz}-4 \mathrm{~Hz})$. A gain $>1000$ is obtained at the main SA resonance $(0.3 \mathrm{~Hz})$. The RMS translational motion of the IP (calculated as $x_{\mathrm{RMS}}(f)=\sqrt{\left.\int_{f}^{\infty} \tilde{x}^{2}(\nu) \mathrm{d} \nu\right)}$ in 10 sec. is reduced from more than 30 to $0.3 \mu \mathrm{m}$. The closed loop floor noise corresponds to the fraction of seismic noise reinjected by using the position sensors for the DC control and can, in principle, be reduced by a steeper low pass filtering of the LVDT signal at $f>f_{\text {merge }}$ and by lowering $f_{\text {merge }}$ : both this solution have drawbacks and need a careful implementation. 

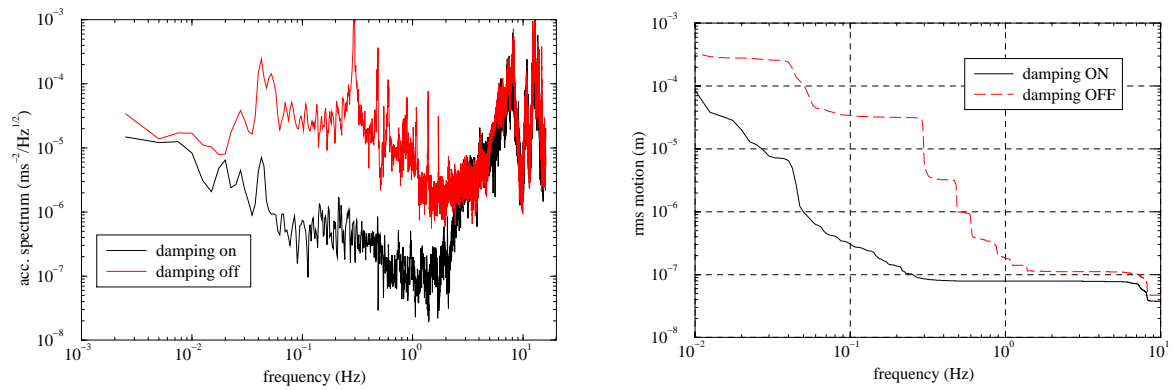

Figure 9. Performance of the inertial control $(X, Y, \Theta$ loops closed $)$ of the superattuenuator, measured on the top of the IP: the left plot shows the acceleration spectral density as measured by the virtual accelerometer $X$ (translation). The right plot shows the effect of the feedback on the RMS residual motion of the IP as a function of the frequency.

Preliminary measurements of the displacement of the mirror with respect to ground have been performed in air, using an LVDT position sensor. The residual RMS mirror motion in $10 \mathrm{sec}$. is $\mathrm{F}$ :

$$
x_{\mathrm{RMS}}(0.1 \mathrm{~Hz}) \leq 3 \mu \mathrm{m} .
$$

When the damping is on such a measurement can provide only an upper bound because the LVDT output is dominated by the seismic motion of the ground.

\section{Conclusions}

In this lecture we have tried to outline how to face the problem of reducing the free motion of the suspended optical components of the VIRGO interferometer, associated to the resonances of the suspension. This is only the beginning: once the motion of the mirrors is reduced to a few microns, the lower control stages can operate to lock the interferometer in the correct operation state.

\section{Acknowledgments}

The author wishes to thank all the people of the Pisa and Florence VIRGO Groups. Among them, special thanks to Diego Passuello, Alberto Gennai and

${ }^{c}$ This number has been obtained with a simpler feedback design, less aggressive than the one of fig. B: the gain raised as $1 / f$ (pure viscous damping force), the crossover frequency was $30 \mathrm{mHz}$ and no compensation of the dips was needed. 
Andrea Marin.

\section{References}

1. P.R.Saulson, Fundamentals of interferometric gravitational wave detectors, World Scientific (1994).

2. G.Losurdo for Pisa VIRGO Group, Proc. of the Second E.Amaldi Conference on Gravitational Waves, CERN, Geneva, 1-4 July 1997, eds. E.Coccia, G.Pizzella, G.Veneziano, World Scientific (1998).

3. F.Frasconi, et al., Performances of the R\&D superattenuator chain of the VIRGO experiment, to be published in the proceedings of the XXXIVth Rencontres de Moriond (1999).

4. A.Giazotto, this volume.

5. G.Losurdo, et al., Rev. Sci. Instrum., 70 (5), 2507-2515 (1999).

6. S.Braccini, et al., Rev. Sci. Instrum., 66 (3), 2672-2676 (1995).

7. S.J.Richman, J.A.Giaime, D.B.Newell, R.T.Stebbins, P.L.Bender, J.E.Faller, Rev. Sci. Instrum., 69 (6), 2531 (1998).

8. A.Gennai, et al., A control model for the inverted pendulum, VIRGO Note VIR-TRE-PIS-4900-102 (1997).

9. G.Losurdo, Ultra-low frequency inverted pendulum pre-isolator for the VIRGO test mass suspension, PhD Thesis, Scuola Normale Superiore, Pisa (1998).

10. G.Losurdo, in the Proceedings of the Third E.Amaldi Conference on Gravitational Waves Experiment, Caltech, Pasadena, 12-16 July 1999, gr-qc/9911044 (1999).

11. A.Gennai, G.Losurdo, A.Marin, D.Passuello, Inertial damping of the superattenuator, VIRGO Note VIR-TRE-PIS-4900-104 (1999). 